\title{
Global and regional burden of stroke during 1990-2010: findings from the Global Burden of Disease Study 2010
}

\author{
Valery L Feigin, Mohammad H Forouzanfar, Rita Krishnamurthi, George A Mensah, Myles \\ Connor, Derrick A Bennett, Andrew E Moran, Ralph L Sacco, Laurie Anderson, Thomas \\ Truelsen, Martin O'Donnell, Narayanaswamy Venketasubramanian, Suzanne Barker-Collo, \\ Carlene M M Lawes, Wenzhi Wang, Yukito Shinohara, Emma Witt, Majid Ezzati, Mohsen \\ Naghavi, and Christopher Murray ${ }^{\star}$ on behalf of the Global Burden of Diseases, Injuries, and \\ Risk Factors Study 2010 (GBD 2010) and the GBD Stroke Experts Group \\ National Institute for Stroke and Applied Neurosciences, Faculty of Health and Environmental \\ Studies, Auckland Uwniversity of Technology, Auckland, New Zealand (Prof V L Feigin MD, R \\ Krishnamurthi PhD, E Witt MSc); Institute for Health Metrics and Evaluation, Department of \\ Global Health, University of Washington, Seattle, WA, USA (Prof M H Forouzanfar MD, Prof M \\ Naghavi MD, Prof C Murray MD); National Institutes of Health Heart, Lung, and Blood Institute, \\ Bethesda, MD, USA (G A Mensah, MD); Consultant Neurologist, National Health Service \\ Borders, Melrose, UK (M Connor MBBCh); Division of Clinical Neurosciences, University of \\ Edinburgh, UK (M Connor); Bute Medical School, University of St Andrews, UK (M Connor); \\ School of Public Health, University of the Witwatersrand, South Africa (M Connor); Clinical Trial \\ Service Unit and Epidemiological Studies Unit, Nuffield Department of Population Health, \\ University of Oxford, Oxford, UK (D A Bennett PhD); Division of General Medicine, Columbia \\ University Medical Center, NY, USA (Prof A E Moran MD); Miller School of Medicine, University \\ of Miami, Miami, FL, USA (Prof R L Sacco MD); Department of Epidemiology, School of Public \\ Health, University of Washington, and Washington State Institute for Public Policy, WA, USA \\ (Prof L M Anderson); Department of Neurology, Copenhagen University Hospital Herlev, Herlev, \\ Denmark (T Truelsen MD); MRC-HPA Centre for Environment and Health, Department of \\ Epidemiology and Biostatistics, School of Public Health, Imperial College London, London, UK \\ (Prof M Ezzati PhD); National University of Ireland, Galway, Ireland (Prof M O'Donnell PhD); \\ Division of Neurology, University Medicine Cluster, Yong Loo Lin School of Medicine, and Saw \\ Swee Hock School of Public Health, National University of Singapore, Singapore (N \\ Venketasubramanian MBBS); School of Psychology (Prof S Barker-Collo PhD) and National \\ Institute for Health Innovation (C M M Lawes MBChB), University of Auckland, Auckland, New
}

Correspondence to: Prof Valery L Feigin, National Institute for Stroke and Applied Neurosciences, School of Rehabilitation and Occupation Studies, School of Public Health and Psychosocial Studies, Faculty of Health and Environmental Studies, Auckland University of Technology University, Auckland 1142, New Zealand, valery.feigin@aut.ac.nz.

Members of the GBD Stroke Expert Group listed at end of paper

Contributors

VLF, AEM, GM, and ME developed the study concept and oversaw the research. VLF, RK, EW, LA, MC, DB undertook reviews of studies. VLF, RK, EM, and SB-C extracted data from retrieved articles and, together with MHF and MN, managed databases. MHF and MN undertook statistical analysis of the data and produced graphs. VLF wrote the first draft of the report. MHF, MN, and CM developed the statistical model and wrote a section on statistical analysis and DALYs estimates. All authors contributed to the critical revision of the manuscript for important intellectual content.

Conflicts of interest

We declare that we have no conflicts of interest. 
Zealand; Beijing Neurosurgical Institute, Capital Medical University, Beijing, China (Prof W Wang PhD); and Federation of National Public Service Personnel Mutual Aid Associations Tachikawa Hospital, Tachikawa, Tokyo, Japan (Y Shinohara, MD)

\section{Summary}

Background-Although stroke is the second leading cause of death worldwide, no comprehensive and comparable assessment of incidence, prevalence, mortality, disability, and epidemiological trends has been estimated for most regions. We used data from the Global Burden of Diseases, Injuries, and Risk Factors Study 2010 (GBD 2010) to estimate the global and regional burden of stroke during 1990-2010.

Methods-We searched Medline, Embase, LILACS, Scopus, PubMed, Science Direct, Global Health Database, the WHO library, and WHO regional databases from 1990 to 2012 to identify relevant studies published between 1990 and 2010. We applied the GBD 2010 analytical technique (DisMod-MR), based on disease-specific, pre-specified associations between incidence, prevalence, and mortality, to calculate regional and country-specific estimates of stroke incidence, prevalence, mortality, and disability-adjusted life-years (DALYs) lost by age group ( $<75$ years, $\geq 75$ years, and in total) and country income level (high-income, and low-income and middleincome) for 1990, 2005, and 2010.

Findings-We included 119 studies (58 from high-income countries and 61 from low-income and middle-income countries). From 1990 to 2010, the age-standardised incidence of stroke significantly decreased by $12 \%$ (95\% CI 6-17) in high-income countries, and increased by $12 \%$ (3 to 22) in low-income and middle-income countries, albeit non-significantly. Mortality rates decreased significantly in both high income (37\%, 31-41) and low-income and middle-income countries $(20 \%, 15-30)$. In 2010, the absolute numbers of people with first stroke (16.9 million), stroke survivors (33 million), stroke-related deaths (5.9 million), and DALYs lost (102 million) were high and had significantly increased since 1990 (68\%, 84\%, 26\%, and 12\% increase, respectively), with most of the burden (68.6\% incident strokes, $52.2 \%$ prevalent strokes, $70.9 \%$ stroke deaths, and 77.7\% DALYs lost) in low-income and middle-income countries. In 2010, $5 \cdot 2$ million (31\%) strokes were in children (aged <20 years old) and young and middle-aged adults (20-64 years), to which children and young and middle-aged adults from low-income and middleincome countries contributed almost 74000 (89\%) and 4.0 million (78\%), respectively, of the burden. Additionally, we noted significant geographical differences of between three and ten times in stroke burden between GBD regions and countries. More than $62 \%$ of new strokes, $69.8 \%$ of prevalent strokes, $45.5 \%$ of deaths from stroke, and $71.7 \%$ of DALYs lost because of stroke were in people younger than 75 years.

Interpretation-Although age-standardised rates of stroke mortality have decreased worldwide in the past two decades, the absolute number of people who have a stroke every year, stroke survivors, related deaths, and the overall global burden of stroke (DALYs lost) are great and increasing. Further study is needed to improve understanding of stroke determinants and burden worldwide, and to establish causes of disparities and changes in trends in stroke burden between countries of different income levels.

Funding-Bill \& Melinda Gates Foundation. 


\section{Introduction}

Estimates from the Global Burden of Diseases, Injuries, and Risk Factors Study (GBD 2010) ranked stroke as the second most common cause of death ${ }^{1}$ and the third most common cause of disability-adjusted life-years (DALYs) ${ }^{2}$ worldwide in 2010. A previous systematic review $^{3}$ of population-based studies of stroke incidence from 28 countries showed that incidence is increasing in low-income and middle-income countries, by contrast with highincome countries where a $42 \%$ decrease in incidence has taken place in the past four decades. However, no comprehensive and comparable assessment of stroke incidence, prevalence, mortality, disability burden (as measured by DALYs lost), and recent epidemiologic trends has been estimated for most world regions. The GBD 2010 investigators developed statistical methods ${ }^{4}$ to address incomplete epidemiological data and provide the most complete and com parable estimates of the global burden of 291 diseases and injuries in all countries grouped into 21 world regions ( ${ }^{\text {appendix }}$ ). We report estimates from GBD 2010 for stroke incidence, mortality, prevalence, and DALYs lost in all 21 GBD world regions in 1990, 2005, and 2010.

\section{Methods}

\section{Literature search and data extraction}

Details of study eligibility criteria, literature search strategy, and data extraction have been described in detail elsewhere. ${ }^{4,5}$ In brief, we included only studies that used WHO's definition of stroke, ${ }^{6}$ that reported methods for ascertainment stroke cases, that distinguished between first-ever and recurrent stroke (only incident strokes were included in these analyses), and that reported an age-specific epidemiological parameter of interest and the population denominator (ie, stroke incidence or prevalence in 5 year or 10 year age bands) with sufficient detail to enable an estimate of age-adjusted variables. Incidence studies from high-income countries had to include complete ascertainment of stroke cases (ie, admitted or not admitted to hospital, fatal and non-fatal) with several overlapping sources of information (including family physicians and other community health services, nursing homes, hospital admissions and discharges, neuroimaging and rehabilitation services, and death certificates) as specified for ideal population-based studies. ${ }^{7} \mathrm{We}$ allowed less rigorous case ascertainment for studies from low-income and middle-income countries in which no other relevant data were otherwise available (appendix $) .5$

\section{Stroke death and disease modelling}

We used the GBD 2010 mortality database and an ensemble cause-of-death modelling approach to estimate the mortality rate. ${ }^{1,8}$ The appendix shows the number of datapoints by decade for each data source. A key element of the analysis of cause-of-death data is to take the raw cause of death data and enhance comparability by mapping across various revisions and national variants of the International Classification of Diseases, and to redistribute socalled garbage codes to valid cause-of-death codes (appendix). ${ }^{8}$ We used the Cause of Death Ensemble model (CODEm) for analysis of death data to estimate age-specific mortality by

See Online for appendix 
country for each stroke type. ${ }^{8,9} \mathrm{We}$ selected a set of relevant covariates and assumed a plausible direction of effect on the basis of existing scientific literature ( ${ }^{\text {appendix }}$ ). A primary objective of the analysis of cause of death was to provide estimates for countries and years that had few data. To inform this analysis, we used several country-level covariates with the constraint of a resultant plausible effect on outcome. We kept a risk factor (eg, blood pressure) in the model if it had a positive association with death, and a protective factor (eg, omega-3 consumption) if it had a negative association (plausible direction). We assumed that a hierarchy of risk existed that would be retained in the model. As such, we gave risk factors with a strong pathophysiological effect (level 1 -ie, the top-most effect) precedence over less strong risk factors and factors that were not directly related, such as country income per person, education, and war.

The ensemble approach combined estimates from several best-performing individual models made with different combinations of covariates and statistical approaches. ${ }^{9}$ We used six different modelling strategies to estimate stroke mortality dependent on the strength of the available data. All these strategies were designed to generate uncertainty distributions for rates of stroke death. The model of stroke mortality was an ensemble of selected singlecause fraction models. In a separate process-the CODCorrect process-the number of deaths was rescaled to total the overall number of deaths (mortality envelope) for a country, age, and sex. This process ensured that the sum of deaths from cardiovascular disease would be equal to all deaths from this disease (modelled independently). The appendix shows the estimates before and after the correction for stroke death.

\section{DALYs}

DALYs have two components: years of life lost (YLL) because of death and years lived with disability (YLD). The methodology for calculation of DALYs in GBD 2010 has been described elsewhere. ${ }^{2,4}$ Briefly, to calculate YLL, the age at death for each fatality is subtracted from the reference life expectancy at that age. ${ }^{10}$ YLD represent healthy life-years lost in survivors, and are calculated from the number of patients living with stroke sequelae, the number of years living with disability due to the disease, and the disability weight (ie, the severity of the disease state). Disability weights range between 0 and 1 , whereby 0.0 denotes no disability (symptom, limitation, or loss of health), and 1.0 denotes a life value equal to death. ${ }^{2}$ To calculate YLD, the stroke prevalence is multiplied by the disability weight. To control for comorbidity, we estimated YLD with a microsimulation method assuming independent probabilities of comorbid diseases (eg, stroke plus diabetes). The resulting disability weights for comorbidity matched the pattern of quality of life in patients with comorbid illnesses in the US Medical Expenditure Panel Survey (MEPS) study ${ }^{2}$ adjusted by decreasing of total disability (total disability of two sequelae in a person would be less than the sum of each disability). The disability weights were calculated with personal interviews in Bangladesh, Indonesia, Peru, and Tanzania; telephone interviews in the USA; and an open-access web-based survey. ${ }^{11}$ Five sequelae of stroke were defined and assessed for GBD. The appendix presents lay descriptions for disability of each stroke sequela with the associated disability weights used in GBD 2004. 
The second important factor is distribution of severity or levels of disability within a disorder. We empirically identified the distribution of severity for chronic stroke by analysis of individual-level measurements of functional health status with the 12-item short form questionnaire for three population surveys: the US MEPS 2000-2009, the National Epidemiological Survey on Alcohol and Related Conditions (NESARC) 2000-2001 and 2004-2005, and the Australian National Survey of Mental Health and Wellbeing of Adults (AHS) 1997. ${ }^{4,10,12} \mathrm{We}$ calculated the severity distribution for acute stroke with findings from studies of the distribution of functional disability immediately ( $₫$ days after the event) and after (within the first month) stroke measured by the Rankin Scale. ${ }^{13}$ We assumed the same disability for haemorrhagic and ischaemic stroke during the hyperacute phase (ie, 1-3 days after the event), but considered disability separately for these stroke types after day three. We assumed a similar distribution of severity on the basis of the GBD sequela definition and Rankin Scale categories. ${ }^{14}$ A slight difference between the definition of disability and categories in GBD 2010 and the Rankin Scale ${ }^{13}$ was regarded as acceptable by consensus of members of an international collaborative stroke expert group (appendix). We calculated a weighted average of disability to estimate a disability weight for 28 days after acute stroke of 0.1 for the first 3 days after stroke and 0.9 after 3 days. These data are a heterogeneous set from developed and developing countries. Scientific literature about the functional status of acute patients that could be translated for GBD purposes was scarce; we therefore included all available information.

\section{Stroke incidence and prevalence}

Stroke survivors have two sequelae: acute stroke and chronic stroke. For each sequela, we used models to estimate the number of patients in each age group, sex, region, and year. The appendix presents the studies identified in a systematic review of the literature. Most studies reported incidence of first-ever stroke. In GBD, sequelae of acute stroke includes all cases, including first-ever and repeated events. We used meta-regression weighted by the proportion of first-ever stroke in all cases of acute stroke to pool the results of studies from The Netherlands, ${ }^{15}$ Spain,,${ }^{16}$ and Australia. ${ }^{17}$ These studies reported both all-stroke and firstever stroke incidence by age. We used the same proportion of first-ever stroke to all stroke for all GBD regions. We defined a patient with chronic stroke as being diagnosed by a physician or having a history of definite stroke. Data for stroke prevalence include all stroke patients irrespective of the type of acute stroke. To be consistent with prevalence and mortality data of chronic stroke, we calculated incidence of chronic stroke (stroke survivors). We multiplied incidence of first-ever stroke by survival proportion after 28 days to estimate incidence of chronic stroke at the regional level. We used the GBD 2010 analytical technique DisMod-MR to estimate the number of incident strokes overall and ischaemic and haemorrhagic strokes (non-ischaemic; prevalence estimates were for total strokes only).

To estimate prevalence of acute stroke cases, we modelled incidence and case fatality of acute strokes. An incident case is a person who has been diagnosed with the type of stroke or who has died due to stroke. By definition, a prevalent case would be a person having a stroke in the past 28 days. Finally, because generally more data were available for incidence of total stroke, we used this aspect to improve stroke-specific incidence by rescaling the 
results of stroke by type to be summed to all-stroke incidence. We corrected the estimated incidence and prevalence on the basis of the proportion of first-ever stroke in incidence of total stroke to identify the prevalence of all-stroke cases of acute stroke (first-ever and recurrent strokes; ${ }^{\text {appendix }}$ ). We also calculated mortality-to-incidence ratio for each region and country as an indicator of the success or failure of strategies for stroke management in a specific region or country (ratio numbers were based on the total number of incident cases and deaths). We estimated $\mathrm{p}$ values on the basis of 1000 draws of the posterior distribution of each statistic. Because some posterior distributions deviated significantly from normal distribution, 2.5 and 97.5 percentiles of the draws were reported as lower and upper bounds of the uncertainty interval for the statistic. We calculated $95 \%$ CIs for all rates.

We analysed stroke incidence, prevalence, and mortality rates for 21 GBD regions that we combined into two major categories: high-income countries (North America, western and central Europe, Australasia, and high-income Asia Pacific) and low-income and middleincome countries (central, east, south, and southeast Asia; eastern Europe; Andean, central, southern, and tropical Latin America and the Caribbean; and North Africa and the Middle East, Oceania, central, east, southern, and west sub-Saharan Africa). We present overall data for stroke burden by country income level. We report age-standardised incidence and mortality rates per 100000 person-years, and prevalence and DALYs estimates per 100000 people with the direct method of standardisation and WHOs standard population as a reference. ${ }^{18}$

\section{Role of the funding source}

The sponsor of the study had no role in study design, data collection, data analysis, data interpretation, or writing of the report. The Writing and GBD Global Analysis Group had full access to all the data in the study and had final responsibility for the decision to submit for publication.

\section{Results}

The appendix shows a flowchart for selection of studies. Of 16558 potentially eligible studies undertaken in 1990-2010, we selected 119 articles for final analysis: 58 from highincome countries and 61 from low-income and middle-income countries. 59 studies reported incidence and excess mortality data, 34 reported prevalence, and 26 reported data only for excess mortality. The appendix provides a list of studies included in the analysis. Studies of a high methodological quality (quality rating 4-5) constituted 55 (95\%) of the studies in high-income countries and 14 (23\%) of those in low-income and middle-income countries. 105 studies were done in 1990-2004 and 14 in 2005-10; some studies overlapped (200405).

The appendix shows country-specific stroke burden in 1990, 2005, and 2010. We noted significant geographical variations in stroke incidence by country, ranging in 2010 from 60 cases (Kuwait) to 504 cases (Lithuania) per 100000 person-years; mortality rates, ranging from 27 cases (France) to 264 cases (Afghanistan) per 100000 person-years; prevalence, ranging from 82 stroke survivors (Burundi) to 1187 survivors (Canada) per 100000 people; DALYs lost, ranging from 398 (Australia) to 5227 (Afghanistan) per 100000 people; and in 
mortality-to-incidence ratios, ranging from $0 \cdot 11$ (Iran) to $1 \cdot 35$ (Afghanistan). Overall, in 2010, an estimated 16.9 million cases of incident stroke took place worldwide (69\% in lowincome and middle-income countries), 33.0 million prevalent stroke cases (52\% in lowincome and middle-income countries), 5.9 million stroke deaths (71\% in low-income and middle-income countries), and 102.2 million DALYs lost (78\% in low-income and middleincome countries; table 1). More than $38 \%$ of new strokes (50\% in high-income countries and $32 \%$ in low-income and middle-income countries), $30 \%$ of prevalent strokes (40\% in high-income countries and $22 \%$ in low-income and middle-income countries), $55 \%$ of stroke deaths (73\% in high-income countries and $47 \%$ in low-income and middle-income countries), and $28 \%$ of DALYs lost (46\% in high-income countries and $23 \%$ in low-income and middle-income countries) were in people aged 75 years and older (table 1).

Globally, in 2010, the mortality-to-incidence ratio was 0.35 ( 0.32 in high-income countries and 0.36 in low-income and middle-income countries; table 1). Although overall we noted no significant change in age-standardised incidence of stroke between 1990 and 2010 (table 1), the direction of changes was different between countries by income level (a 12\% [95\% CI 6\%-17\%] statistically significant decrease in high-income countries, and a $12 \%$ [ -3 to 22] non-significant increase in low-income and middle-income countries). Further more, there was a significant $25 \%$ reduction in mortality rate (37\% [31-41] in high-income countries and 20\% [15-30] in low-income and middle-income countries), DALYs lost (36\% [30-40] and 22\% [18-32], respectively), and mortality-to-incidence ratio (23\% [14-29] and $27 \%$ [14-38]). Stroke prevalence increased significantly by $27 \%$ (19-43) in high-income countries only; the $8.5 \%$ ( -13 to 34 ) increase in low-income and middle-income countries was not significant. Globally, for 1990-2010, we noted a 25\% (13-33) significant increase in stroke incidence in people aged 20-64 years, mostly attributable to an 18\% (10-25) significant increase in low-income and middle-income countries.

In the past two decades globally, noticeable increases took place in the absolute numbers of people with incident stroke (a $68 \%$ increase), stroke survivors (84\%), stroke-related deaths (26\%), and DALYs lost (12\%; table 1). The most striking increases in the number of stroke survivors (113\%), DALYs lost (31\%), and stroke-related deaths (36\%) were in people aged 75 years and older (table 1). Presently, age-standardised rates of stroke incidence in lowincome and middle-income countries exceed those in high-income countries by $23 \%$ (24\% in people younger than 75 years and $21 \%$ in people aged 75 years and older), but the number of people younger than 75 years with incident stroke in low-income and middle-income countries is more than three times that in high-income countries (table 1). Similarly, the number of DALYs lost in people younger than 75 years in low-income and middle-income countries exceeded those lost in high-income countries by almost five times (table 1), whereas in people aged 75 years and older, DALYs lost in low-income and middle-income countries exceeded those lost in high-income countries by less than two times (table 1). Conversely, the number of stroke survivors aged 75 years and older in high-income countries exceeded the number in low-income and middle-income countries by $40 \%$ (table 1), whereas there were almost $30 \%$ more survivors younger than 75 years in low-income and middle-income countries than in high-income countries (table 1). Age-standardised rates of stroke mortality in people aged 75 years and older in low-income and middle-income countries exceeded mortality rates by $33 \%$ in the same age group of people living in high- 
income countries, and by $212 \%$ for the younger than 75 years population (table 1 ). Mortality to incidence ratios in people younger than 75 years in low-income and middle-income countries were significantly higher at $37 \%$ greater than those in high-income countries, but in those aged 75 years and older, the excess was only 12\% (table 1). In 2010, $5 \cdot 2$ million (31\%) strokes were in children (aged <20 years) and young and middle-aged adults (20-64 years), to which children and young to middle-age adults in low-income and middle-income countries contributed almost $74000(89 \%)$ and 4.0 million (78\%), respectively, of the burden.

Important differences in trends were found between high-income and low-income and middle-income regions in age-adjusted incidence and mortality rates, prevalence, DALYs lost, and mortality-to-ratios by age group (table 1). In high-income countries, agestandardised rates of stroke incidence were most significantly decreased in people aged 75 years and older ( $16 \%$ vs $9.1 \%$ in younger people; table 1), whereas prevalence increased significantly by a similar proportion (23-25\%) in people of both age groups (table 1). Agestandardised mortality rates in high-income countries significantly decreased by a similar proportion in younger and older people (by 36-39\%; table 1), whereas DALYs lost fell by $36 \%$ in people younger than 75 years and by $63 \%$ in older people (table 1). The greatest reduction in mortality-to-incidence ratios was in people younger than 75 years in highincome countries (32\% decrease) compared with a $21 \%$ reduction in people aged 75 years and older, with the lowest ratios shown in 2010 for both age groups (table 1).

In low-income and middle-income countries, age-standardised incidence and prevalence increased almost equally (9-10\%) in younger and older people, but the increases were not significant (table 1). Most striking were significant reductions in rates of age-standardised stroke mortality (24\%), DALYs lost (24\%), and mortality-to-incidence ratios (32\%) in people younger than 75 years compared with older people $(16 \%, 17 \%$, and $23 \%$ respectively; table 1). In 2010, children and youths (aged <20 years) and young and middleaged adults (20-64 years) constituted $0.5 \%$ and $31 \%$, respectively, of all people with incident stroke ( $0.1 \%$ and $21 \%$, respectively, in high-income countries and $0.6 \%$ and $35 \%$, respectively, in low-income and middle-income countries), $1.1 \%$ and $43 \%$ of all prevalent strokes $(0.5 \%$ and $34 \%$ in high-income countries, and $1.6 \%$ and $51 \%$ in low-income and middle-income countries), and $0.7 \%$ and $22 \%$ of all stroke deaths $(0.05 \%$ and $11 \%$ in highincome countries, and $1 \%$ and $26 \%$ in low-income and middle-income countries).

Although the mean age of people with incident stroke worldwide has not significantly changed in the past two decades (table 2), a significant increase took place in the mean age of incident, prevalent, and fatal strokes in both high-income and low-income and middleincome countries (table 2). The mean age of patients with incident and prevalent stroke in high-income countries was more than 5 years greater than that in low-income and middleincome countries (table 2). The mean age that people had fatal strokes in low-income and middle-income countries was more than 8 years younger than that in high-income countries (table 2).

We noted significant geographical variations in stroke burden by GBD region (figures 1-3 and appendix). Percent change in DALYs lost per 100000 people in GBD regions varied 
from $-50 \cdot 4 \%$ to $-0 \cdot 74 \%$ (figure 4 ). By GBD regions, increases were shown in stroke incidence in sub-Saharan Africa (central, east, southern, and west), the Middle East, southeast Asia, central and Latin America, and south and east Asia, but incidence decreased in high-income Asia Pacific (rates remained very high in eastern Europe; figure 1 and appendix). Mortality increased in sub-Saharan Africa, south Asia, and central and Latin America, but decreased in high-income North America, western and central Europe, north Africa and the Middle East, Australasia, and high-income Asia Pacific (rates remained very high in eastern Europe, and central and east Asia; figure 2 and appendix). For all study periods, stroke prevalence was lowest in sub-Saharan Africa, northern Africa, and the Middle East regions ( ${ }^{a p p e n d i x}$ ). Prevalence increased noticeably in high-income North America (from 495-750 per 100000 people in 1990 to 595-1067 in 2010) and western and eastern Europe, east and southeast Asia, Australasia, and high-income Asia Pacific regions (from 355-495 in 1990 to 394-595 in 2010; appendix). Mortality-to-incidence ratios decreased in all GBD regions, except for eastern Europe, south Asia, and high-income Asia Pacific, where they remained largely unchanged (figure 3 and appendix). The most marked reductions in DALYs lost were in west and central sub-Saharan Africa; eastern Europe; and central, south, and southeast Asia (figure 4 and appendix). Incidence and prevalence increased significantly with age in all regions (appendix), but steeper increases in incidence, mortality, and DALYs lost were shown in low-income and middle-income countries, and a steeper increase in prevalence was shown in high-income countries ( ${ }^{\text {appendix }}$ ). Age-specific incidence, mortality, and DALYs lost in low-income and middle-income countries were significantly greater than those in high-income countries, but age-specific prevalence was significantly greater in high-income countries (appendix).

\section{Discussion}

This study is the first to report the global burden of stroke in terms of incidence, prevalence, mortality, DALYs lost, and mortality-to-incidence ratios across GBD regions and all countries in 1990, 2005, and 2010, and for all age groups of the population. Although the estimates from the GBD 2010 Study showed that ischaemic heart disease and stroke were the two greatest causes of death between 1990 and 2010, 1,8 our analysis provides a more detailed insight into the global burden of stroke in high-income and low-income and middleincome countries in the past two decades. Therefore, our results reinforce the conclusions of the 1990, 2001, and GBD 2010 studies about the growing burden of stroke globally and in low-income and middle-income countries in particular (panel).

Worldwide in 2010, roughly $10 \%$ of the 52769700 deaths $^{1}$ and about $4 \%$ of the 2490385 $000 \mathrm{DALYs}^{2}$ were due to stroke. Our findings show that although stroke mortality rates and mortality-to-incidence ratios have decreased in the past two decades, the global burden of stroke in terms of the absolute number of people affected every year, stroke survivors, related deaths, and DALYs lost are great and increasing, with most of the burden in lowincome and middle-income countries. If these trends in stroke incidence, mortality, and DALYs continue, by 2030 there will be almost 12 million stroke deaths, 70 million stroke survivors, and more than 200 million DALYs lost globally. In line with previous findings, ${ }^{3}$ the similar pattern of decline in stroke incidence and mortality suggests that changes in mortality rates are most likely to be attributable to the corresponding changes in incidence. 
Our study also showed that although the mean age of people with stroke is increasing in countries of both income levels, the proportion of stroke in people younger than 65 years is substantial, especially in low-income and middle-income countries, and more than 83000 children and youths aged 20 years and younger are affected by stroke annually.

Furthermore, we noted a substantial geographical difference in stroke burden by GBD regions and country income level, which is likely to be attributed to differences in the national income per person. ${ }^{19}$ Within high-income countries, good health services and strategies for stroke prevention and care (eg, smoking cessation, control of blood pressure, and acute stroke units) are the most likely explanations for the greater reduction in stroke incidence, mortality, mortality-to-incidence ratios, and DALYs lost; the converse has been shown for low-income and middle-income countries. For example, in these countries, stroke incidence has increased, and mortality-to-incidence ratios and DALYs lost are higher than in high-income countries. Even low-income and middle-income regions with a relatively low stroke incidence and prevalence, such as sub-Saharan Africa, have high stroke mortality rates and a low rate of reduction in DALYs lost. The high and increasing DALYs lost due to stroke are consistent with one of GBD 2010's recurring themes: disability is causing an increasing fraction of the burden of disease as demographics and epidemiology evolve..$^{2,10}$ The increasing prevalence of smoking ${ }^{20}$ and other risk factors ${ }^{21}$ for stroke in the developing world is particularly alarming because, if these trends continue and are left unchecked, further increases in stroke burden will take place in low-income and middle-income countries.

Differences in the prevalence and significance of stroke risk factors, ${ }^{21-23}$ and in the accessibility of health services and level of control of these risk factors, ${ }^{24}$ are likely to account for the large geographical variations in stroke incidence. The significantly greater prevalence of stroke in high-income than in low-income and middle-income countries is associated with an inverse association between prevalence of stroke and stroke mortality and DALYs lost (significantly lower stroke mortality and DALYs lost in high-income $v s$ lowincome and middle-income countries). Together with increased mortality-to-incidence ratios in low-income and middle-income countries, this finding suggests differences in the management of acute stroke as the likely explanation of differences in stroke prevalence, with less adequate stroke management being associated with a greater case fatality and subsequent lower levels of stroke prevalence. Although the reduction in stroke mortality in high-income countries is not surprising because it has been consistently reported in most countries of this income level, ${ }^{25-28}$ our findings of the decrease in mortality in low-income and middle-income countries, although not significant, were unexpected. The sensitivity analyses we undertook to assess the effects of incomplete and poor quality data, and for settings where no data were available, gave similar results, thus showing the robustness of estimates of stroke mortality. Although similar reductions in stroke mortality have taken place in some low-income and middle-income countries, ${ }^{29,30}$ these findings should be confirmed for other countries of this income level. As in a previous study ${ }^{30}$ we did not note a strong parallel between changes in stroke incidence and mortality, suggesting that factors contributing to stroke incidence and mortality or their changes (including level of exposure to those factors) over time might be different. ${ }^{31}$ 
Our study is the first to show that the age-specific burden of stroke in low-income and middle-income countries is significantly greater than that in high-income countries, whereas the age-specific prevalence of stroke is significantly greater in high-income countries. Wide geographical variations in rates of stroke mortality, ${ }^{19,32,33}$ with significantly greater rates of age-adjusted mortality and DALYs lost in low-income and middle-income countries than in high-income countries, ${ }^{19}$ have also been reported elsewhere. Worldwide, ageing of the population, overall population growth, reduced stroke case-fatality and epidemiological transition in low-income and middle-income countries, resulting in increases in the prevalence of stroke risk factors, are likely to be the cause of increases in the number of incident strokes, stroke survivors, and DALYs lost. Difficulty in access to health care, and restricted resources in terms of adequate acute stroke treatment, rehabilitation, and secondary stroke prevention (eg, anticoagulation for patients with ischaemic stroke with atrial fibrillation) are likely to account for higher stroke mortality, mortality-to-incidence ratios, and DALYs lost, and lower stroke prevalence in low-income and middle-income countries than in high-income countries. ${ }^{19,34}$

Stroke was traditionally thought of as a disease of elderly people; ${ }^{3}$ however, our data show that the proportion of stroke burden is greater overall in individuals younger than 75 years than in those who are older, especially in low-income and middle-income regions. Although in the past two decades there was a trend towards occurrence of incident stroke later in life, probably because of an ageing population, ${ }^{35}$ the pro portion of young people (aged $<20$ years) and young and middle-aged adults (20-64 years) affected by stroke increased. In addition to a significant increase in the incidence of stroke in young and middle-aged adults, these findings suggest that stroke should no longer be regarded as a disease of old age. A worrisome trend towards increasing stroke incidence in young adults has been reported for some countries. ${ }^{36,37}$ In view of the worldwide epidemic of diabetes ${ }^{38}$ and increasing prevalence of other cardiovascular risk factors in young adults ${ }^{39,40}$ and overall, ${ }^{41-45}$ especially in low-income and middle-income countries, the shift in stroke burden towards younger populations is likely to continue globally unless effective preventive strategies are urgently implemented.

This study was comprehensive in its global coverage, is based on the largest stroke epidemiological dataset to date, and is the first to provide systematic regional and countryspecific estimates of stroke burden; however, it has some limitations. The main limitations include a scarcity of high-quality epidemiological stroke data from low-income and middleincome countries, especially for stroke incidence and mortality. As such, we allowed studies of both high and lower methodological quality from these countries, rather than only studies of high methodological quality as for high-income countries. Additionally, there was substantial regional heterogeneity of the stroke data, particularly that from low-income and middle-income countries. Insufficient high-quality data from low-income and middleincome countries might account for some of the differences noted in the epidemiological characteristics of stroke (eg, the lower mean age at stroke in low-income and middle-income countries than in high-income countries might be related to better ascertainment and diagnostic coding of events at older ages in high-income countries) and stroke burden between countries of different income levels. However, this limitation should not have a marked effect on our estimates because we applied standard methodological criteria for 
selection of studies across the 20 year study period and believe that the quality of data was consistent for included studies.

Stroke diagnosis is unlikely to have changed between 1990 and 2010 because most diagnoses have been based on clinical (WHO) criteria and, when imaging was available, for verification of pathological subtypes on CT images. The consistency of our estimates of stroke incidence, prevalence, and mortality-to-incidence ratios in both low-income and middle-income and high-income countries with those reported in two previous systematic reviews based on methodologically more rigorous ideal population-based studies of stroke incidence and prevalence ${ }^{3,46}$ corroborates our findings. Another limitation is that GBD stroke estimates are based on clinical stroke cases and used the Rankin Scale to index disability weights. There fore, we probably underestimated the burden of stroke by not including so-called silent strokes and vascular dementia in the case definition nor the effect of accelerated long-term cognitive functional decline in our estimation of disability.

Although we cannot exclude the possibility of errors in calculations of stroke disability weights, the high correlation of the weights across settings suggests that a broadly shared set of common values exists for health losses due to stroke, thus increasing our confidence in the reliability of our calculations of disability weights.

New well-designed, comparable, community-based stroke epidemiology and surveillance studies are greatly needed. ${ }^{3,19,47}$ Not only will findings from these studies improve our understanding of stroke determinants and burden worldwide, and establish causes of disparities and changes in trends in stroke burden between countries of different income levels, but they will also help with the development and monitoring of the effectiveness of stroke prevention and management and rehabilitation strategies in different countries and populations. These strategies should be culturally acceptable, affordable, and aimed at both high-risk individuals and the global population, wwith the priority placed on populationbased preventive strategies ${ }^{45}$ to reduce blood pressure and promote smoking cessation. ${ }^{47-49}$ In view of the increasing prevalence of stroke and DALYs lost because of stroke, and the already overstretched health resources worldwide, health-care services need to pay more attention to provision of stroke chronic care (including prevention of secondary stroke) ${ }^{50}$ and should incorporate community rehabilitation, including self-managed rehabilitation strategies.

\section{Acknowledgments}

This work was undertaken as a part of the Global Burden of Diseases, Injuries, and Risk Factors 2010 (GBD 2010) Study. The results in this paper are prepared independently of the final estimates of the GBD 2010 study. We thank Sarah Safranek (University of Washington Health Sciences Library) for her help in developing the systematic review literature search strategies; Michael F MacIntyre, Brittany Wurtz, and Summer Ohno (University of Washington Institute for Health Metrics and Evaluation) for research coordination; Ryan M Barber (University of Washington Institute for Health Metrics and Evaluation) for creating figures and tables for the paper; Hannah Gardener (University of Miami) for helping reviewing some studies; and Helen McDonald (National Institute for Stroke And Applied Neurosciences, Aukland University of Technology) for organising teleconferences and secretarial support. The authors alone are responsible for the views expressed in this publication. 


\title{
Global Burden of Disease Stroke Expert Group members
}

\author{
Laurie Anderson (USA), Suzanne Barker-Collo (New Zealand), Derrick Bennett (UK), \\ Myles Connor (South Africa), Majiid Ezzati (UK), Valery L Feigin (Chairman; New \\ Zealand), Mohammed Forouzanfar (USA), Rita Krishnamurthi (New Zealand), Carlene \\ Lawes (New Zealand), George Mensah (USA), Andrew E Moran (USA), Martin O'Donnell \\ (Ireland), Jeyaraj Durai Pandian (India), Varsha Parag (New Zealand), Ralph Sacco (USA), \\ Yukito Shinohara (Japan), Thomas Truelsen (Denmark), Narayanaswamy \\ Venketasubramanian (Singapore), Emma Witt (New Zealand), Wenzhi Wang (China).
}

\section{References}

1. Lozano R, Naghavi M, Foreman K, et al. Global and regional mortality from 235 causes of death for 20 age groups in 1990 and 2010: a systematic analysis for the Global Burden of Disease Study 2010. Lancet. 2012; 380:2095-2128. [PubMed: 23245604]

2. Murray CJL, Vos T, Lozano R, et al. Disability-adjusted life-years (DALYs) for 291 diseases and injuries in 21 regions, 1990-2010: a systematic analysis for the Global Burden of Disease Study 2010. Lancet. 2012; 380:2197-2223. [PubMed: 23245608]

3. Feigin VL, Lawes CM, Bennett DA, Barker-Collo SL, Parag V. Worldwide stroke incidence and early case fatality reported in 56 population-based studies: a systematic review. Lancet Neurol. 2009; 8:355-369. [PubMed: 19233729]

4. Murray CJL, Ezzati M, Flaxman AD, et al. GBD 2010: design, definitions, and metrics. Lancet. 2012; 380:2063-2066. [PubMed: 23245602]

5. Bennett DA, Anderson LM, Nair N, et al. Methodology of the global and regional burden of stroke study. Neuroepidemiology. 2012; 38:30-40. [PubMed: 22212892]

6. Aho K, Harmsen P, Hatano S, Marquardsen J, Smirnov VE, Strasser T. Cerebrovascular disease in the community: results of a WHO collaborative study. Bull World Health Organ. 1980; 58:113-130. [PubMed: 6966542]

7. Feigin V, Hoorn SV. How to study stroke incidence. Lancet. 2004; 363:1920. [PubMed: 15194247]

8. Wang H, Dwyer-Lindgren L, Lofgren KT, et al. Age-specific and sex-specific mortality in 187 countries, 1970-2010: a systematic analysis for the Global Burden of Disease Study 2010. Lancet. 2012; 380:2071-2094. [PubMed: 23245603]

9. Foreman K, Lozano R, Lopez A, Murray C. Modeling causes of death: an integrated approach using CODEm. Popul Health Metr. 2012; 10:1. [PubMed: 22226226]

10. Vos T, Flaxman AD, Naghavi M, et al. Years lived with disability (YLDs) for 1160 sequelae of 289 diseases and injuries 1990-2010: a systematic analysis for the Global Burden of Disease Study 2010. Lancet. 2012; 380:2163-2196. [PubMed: 23245607]

11. Salomon JA, Vos T, Hogan DR, et al. Common values in assessing health outcomes from disease and injury: disability weights measurement study for the Global Burden of Disease Study 2010. Lancet. 2012; 380:2129-2143. [PubMed: 23245605]

12. Salomon JA, Wang H, Freeman MK, et al. Healthy life expectancy for 187 countries, 1990-2010: a systematic analysis for the Global Burden Disease Study 2010. Lancet. 2012; 380:2144-2162. [PubMed: 23245606]

13. Rankin J. Cerebral vascular accidents in patients over the age of 60: II. Prognosis. Scott Med J. 1957; 2:200-213. [PubMed: 13432835]

14. Bonita R, Beaglehole R. Recovery of motor function after stroke. Stroke. 1988; 19:1497-1500. [PubMed: 3201508]

15. Herman B, Leyten AC, van Luijk JH, Frenken CW, Op de Coul AA, Schulte BP. Epidemiology of stroke in Tilburg, the Netherlands. the population-based stroke incidence register: 2. Incidence, initial clinical picture and medical care, and three-week case fatality. Stroke. 1982; 13:629-634. [PubMed: 7123595] 
16. Alzamora MT, Sorribes M, Heras A, et al. Ischemic stroke incidence in Santa Coloma de Gramenet (ISISCOG), Spain. A community-based study. BMC Neurology. 2008; 8:5. [PubMed: 18371212]

17. Thrift AG, Dewey HM, Macdonell RA, McNeil JJ, Donnan GA. Stroke incidence on the east coast of Australia: the North East Melbourne Stroke Incidence Study (NEMESIS). Stroke. 2000; 31:2087-2092. [PubMed: 10978034]

18. Ahmad, OB.; Boschi-Pinto, C.; Lopez, AD.; Murray, CJL.; Lozano, R.; Inoue, M. Age standardization of rates: a new WHO standard. Luxembourg: World Health Organization; 2013.

19. Johnston SC, Mendis S, Mathers CD. Global variation in stroke burden and mortality: estimates from monitoring, surveillance, and modelling. Lancet Neurol. 2009; 8:345-354. [PubMed: 19233730]

20. WHO. WHO report on the global tobacco epidemic. Luxembourg: World Health Organization; 2013.

21. Lim SS, Vos T, Flaxman AD, et al. A comparative risk assessment of burden of disease and injury attributable to 67 risk factors and risk factor clusters in 21 regions, 1990-2010: a systematic analysis for the Global Burden of Disease Study 2010. Lancet. 2012; 380:2224-2260. [PubMed: 23245609]

22. Putaala J, Yesilot N, Waje-Andreassen U, et al. Demographic and geographic vascular risk factor differences in European young adults with ischemic stroke: the 15 Cities Young Stroke study. Stroke. 2012; 43:2624-2630. [PubMed: 22798330]

23. O’Donnell MJ, Xavier D, Liu L, et al. Risk factors for ischaemic and intracerebral haemorrhagic stroke in 22 countries (the INTERSTROKE study): a case-control study. Lancet. 2010; 376:112123. [PubMed: 20561675]

24. WHO. Global Atlas on Cardiovascular disease prevention and control. Geneva: World Health Organization; 2011.

25. Vaartjes I, O'Flaherty M, Capewell S, Kappelle J, Bots M. Remarkable decline in ischemic stroke mortality is not matched by changes in incidence. Stroke. 2013; 44:591-597. [PubMed: 23212165]

26. Gillum RF, Kwagyan J, Obisesan TO. Ethnic and geographic variation in stroke mortality trends. Stroke. 2011; 42:3294-3296. [PubMed: 21940976]

27. Kunst AE, Amiri M, Janssen F. The decline in stroke mortality: exploration of future trends in 7 western European countries. Stroke. 2011; 42:2126-2130. [PubMed: 21700943]

28. Redon J, Olsen MH, Cooper RS, et al. Stroke mortality and trends from 1990 to 2006 in 39 countries from Europe and Central Asia: implications for control of high blood pressure. Eur Heart J. 2011; 32:1424-1431. [PubMed: 21487117]

29. André C, Curioni CC, Braga da Cunha C, Veras R. Progressive decline in stroke mortality in Brazil from 1980 to 1982, 1990 to 1992, and 2000 to 2002. Stroke. 2006; 37:2784-2789. [PubMed: 17008629]

30. Stringhini S, Sinon F, Didon J, Gedeon J, Paccaud F, Bovet P. Declining stroke and myocardial infarction mortality between 1989 and 2010 in a country of the African region. Stroke. 2012; 43:2283-2288. [PubMed: 22700529]

31. Truelsen T, Mahonen M, Tolonen H, et al. Trends in stroke and coronary heart disease in the WHO MONICA Project. Stroke. 2003; 34:1346-1352. [PubMed: 12738889]

32. Sarti C, Rastenyte D, Cepaitis Z, Tuomilehto J. International trends in mortality from stroke, 1968 to 1994. Stroke. 2000; 31:1588-1601. [PubMed: 10884459]

33. Sarti C, Stegmayr B, Tolonen H, Mahonen M, Tuomilehto J, Asplund K. Are changes in mortality from stroke caused by changes in stroke event rates or case fatality? Results from the WHO MONICA Project. Stroke. 2003; 34:1833-1840. [PubMed: 12855832]

34. Norrving B, Kissela B. The global burden of stroke and need for a continuum of care. Neurology. 2013; 80(suppl):S5-S12. [PubMed: 23319486]

35. Cairncross F. Economics: age, health and wealth. Nature. 2007; 448:875-876. [PubMed: 17713520]

36. Kissela BM, Khoury JC, Alwell K, et al. Age at stroke: temporal trends in stroke incidence in a large, biracial population. Neurology. 2012; 79:1781-1787. [PubMed: 23054237] 
37. Medin J, Nordlund A, Ekberg K. Increasing stroke incidence in Sweden between 1989 and 2000 among persons aged 30 to 65 years: evidence from the Swedish Hospital Discharge Register. Stroke. 2004; 35:1047-1051. [PubMed: 15073400]

38. Danaei G, Finucane MM, Lu Y, et al. National, regional, and global trends in fasting plasma glucose and diabetes prevalence since 1980: systematic analysis of health examination surveys and epidemiological studies with 370 country-years and 2.7 million participants. Lancet. 2011; 378:31-40. [PubMed: 21705069]

39. The Lancet. Challenging age_old ideas about stroke. Lancet Neurol. 2012; 79:1013.

40. de los Rios F, Kleindorfer DO, Khoury J, et al. Trends in substance abuse preceding stroke among young adults: a population-based study. Stroke. 2012; 43:3179-3183. [PubMed: 23160887]

41. Danaei G, Finucane MM, Lin JK, et al. National, regional, and global trends in systolic blood pressure since 1980: systematic analysis of health examination surveys and epidemiological studies with 786 country-years and 5.4 million participants. Lancet. 2011; 377:568-577. [PubMed: 21295844]

42. Farzadfar F, Finucane MM, Danaei G, et al. National, regional, and global trends in serum total cholesterol since 1980: systematic analysis of health examination surveys and epidemiological studies with 321 country-years and 3.0 million participants. Lancet. 2011; 377:578-586. [PubMed: 21295847]

43. Finucane MM, Stevens GA, Cowan MJ, et al. National, regional, and global trends in body-mass index since 1980: systematic analysis of health examination surveys and epidemiological studies with 960 country-years and 9.1 million participants. Lancet. 2011; 377:557-567. [PubMed: 21295846]

44. The Lancet. An epidemic of risk factors for cardiovascular disease. Lancet. 2011; 377:527. [PubMed: 21315931]

45. Anand SS, Yusuf S. Stemming the global tsunami of cardiovascular disease. Lancet. 2011; 377:529-532. [PubMed: 21295845]

46. Feigin VL, Lawes CMM, Bennett DA, Anderson CS. Stroke epidemiology: a review of population-based studies of incidence, prevalence, and case-fatality in the late 20th century. Lancet Neurol. 2003; 2:43-53. [PubMed: 12849300]

47. Sidney S, Rosamond WD, Howard VJ, Luepker RV. The "Heart Disease and Stroke Statistics-2013 Update" and the need for a national cardiovascular surveillance system. Circulation. 2013; 127:21-23. [PubMed: 23239838]

48. Feigin VL, Krishnamurthi R. Stroke prevention in the developing world. Stroke. 2011; 42:36553658. [PubMed: 22052509]

49. Strong K, Mathers C, Bonita R. Preventing stroke: saving lives around the world. Lancet Neurol. 2007; 6:182-187. [PubMed: 17239805]

50. Yusuf S, Islam S, Chow CK, et al. Use of secondary prevention drugs for cardiovascular disease in the community in high-income, middle-income, and low-income countries (the PURE Study): a prospective epidemiological survey. Lancet. 2011; 378:1231-1243. [PubMed: 21872920] 


\section{Panel: Research in context}

\section{Systematic review}

We searched Medline, Embase, LILACS, Scopus, PubMed, Science Direct, Global Health Database, the WHO library, and WHO regional databases between 1990 and 2012 to identify studies published between 1990 and 2010. To be included in this systematic review, studies of stroke incidence or prevalence had to meet several core requirements. First, stroke should have been defined according to the standard WHO clinical criteria as "a rapidly developed sign of focal (or global) disturbance of cerebral function lasting longer than 24 hours (unless interrupted by death), with no apparent nonvascular cause." Incidence and mortality studies from developed countries should have had a complete stroke case ascertainment (hospitalised and non-hospitalised, fatal and non-fatal) using various overlapping sources of information as specified for ideal population-based studies. We allowed less rigorous case ascertainment only for studies from low-income and middle-income countries in which no other relevant data were available otherwise. Second, the study should have distinguished between first-ever stroke and recurrent stroke (only first-ever-in-a-lifetime events were included in the systematic analysis). Third, the study should have reported on an age-specific epidemiologic parameter of interest (ie, stroke incidence, prevalence, mortality, or denominator) sufficient to estimate age-adjusted stroke incidence, mortality, or prevalence.

\section{Interpretation}

This study is the first to report the global burden of stroke in terms of incidence, prevalence, mortality, DALYs lost, and mortality-to-incidence ratios across GBD regions and all countries in 1990, 2005, and 2010, and for all age groups of the population. Our findings show that although stroke mortality rates and mortality-to-incidence ratios have decreased in the past two decades, the global burden of stroke in terms of the absolute number of people affected every year, stroke survivors, related deaths, and DALYs lost are great and increasing, with most the burden in low-income and middle-income countries. If these trends in stroke incidence, mortality, and DALYs continue, by 2030 there will be almost 12 million stroke deaths, 70 million stroke survivors, and more than 200 million DALYs lost globally. New well-designed, comparable, community-based stroke epidemiology and surveillance studies are needed to improve our understanding of stroke determinants and burden worldwide, and to establish causes of disparities and changes in trends in stroke burden between countries of different income levels. Findings from these studies will also help with the development and monitoring of the effectiveness of stroke prevention and management and rehabilitation strategies in different countries and populations. 


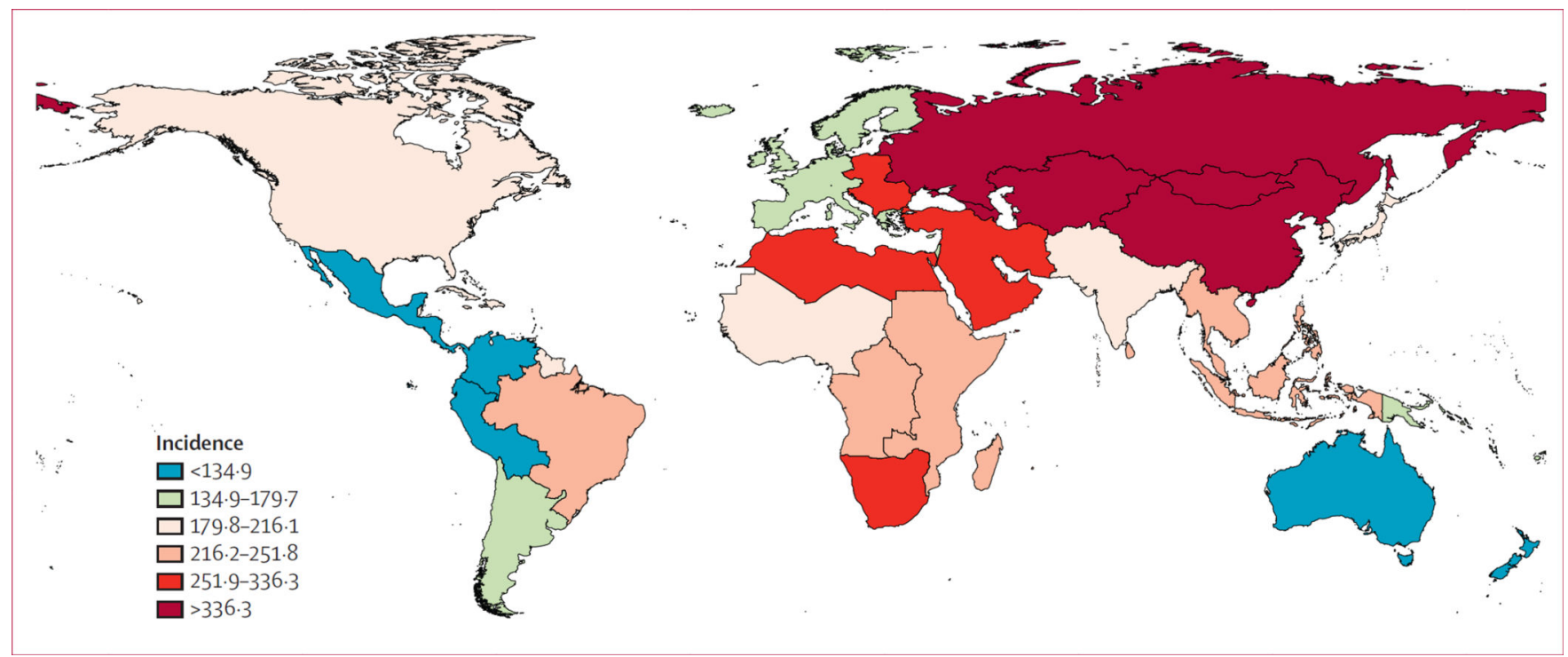

Figure 1.

Age-standardised stroke incidence per 100000 person-years for 2010 


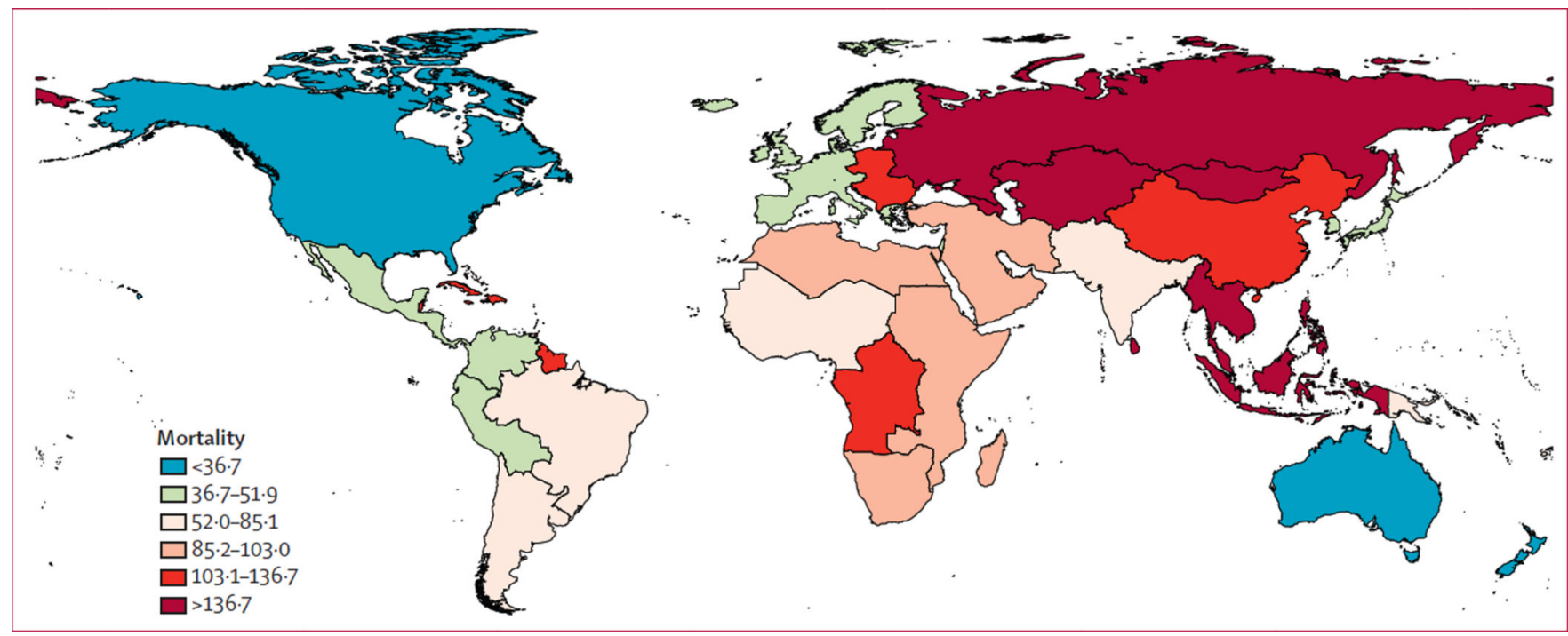

Figure 2.

Age-standardised stroke mortality per 100000 people for 2010 


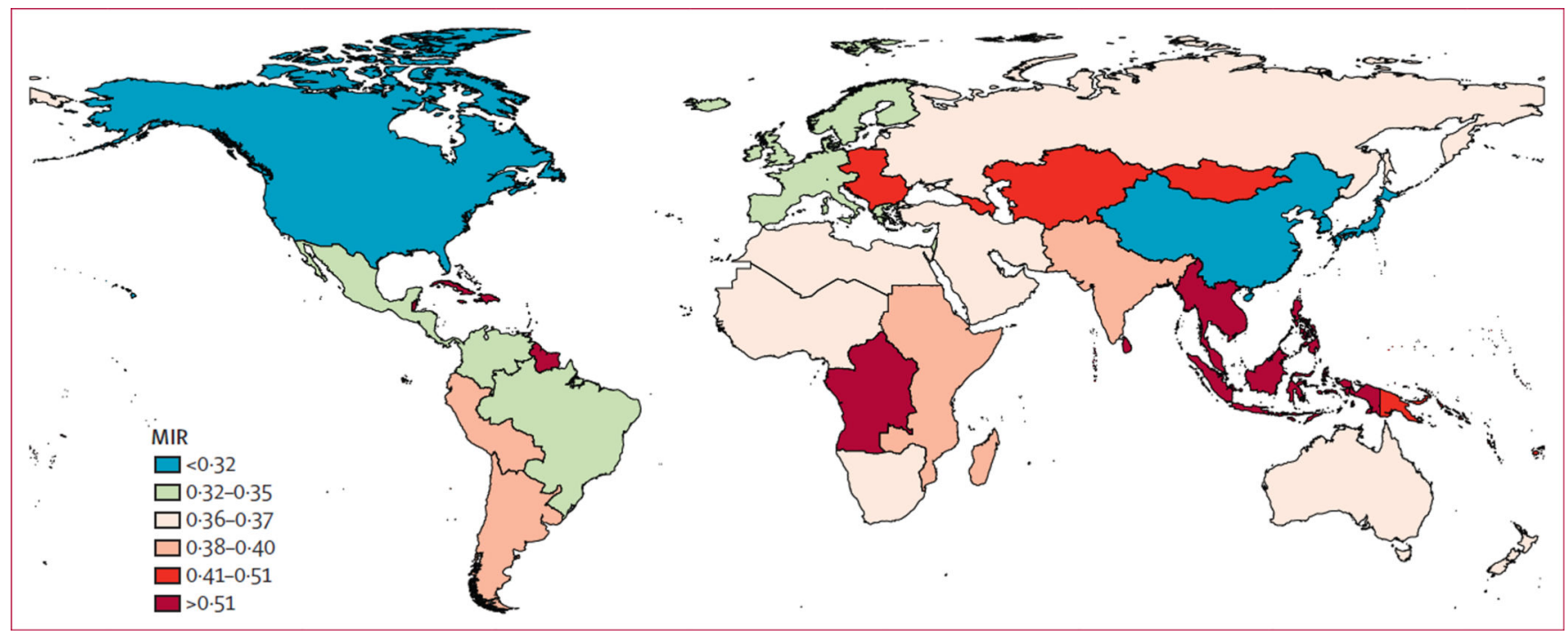

Figure 3.

Stroke mortality-to-incidence ratio (MIR) for 2010 


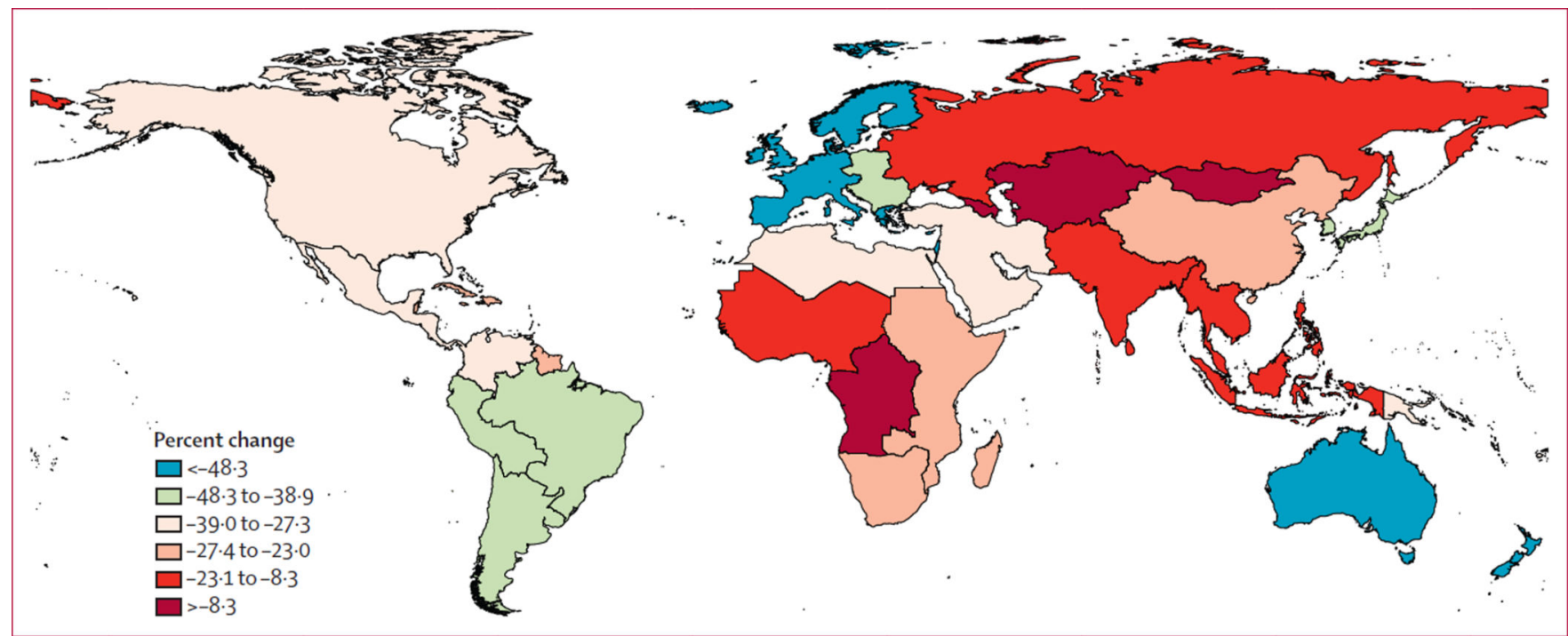

Figure 4.

Percent change in age-standardised disability-adjusted life-years for stroke per 100000 people for 2010 


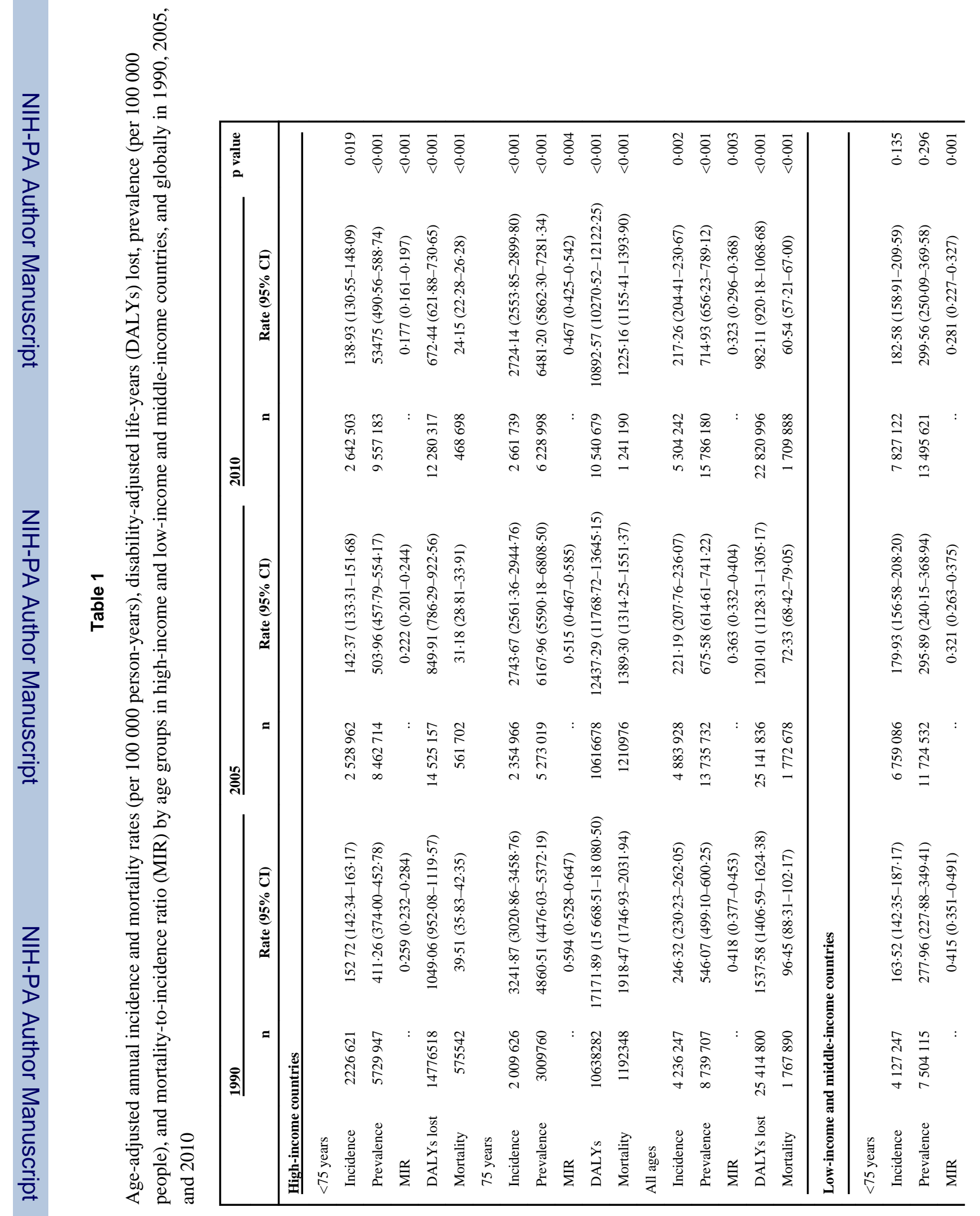




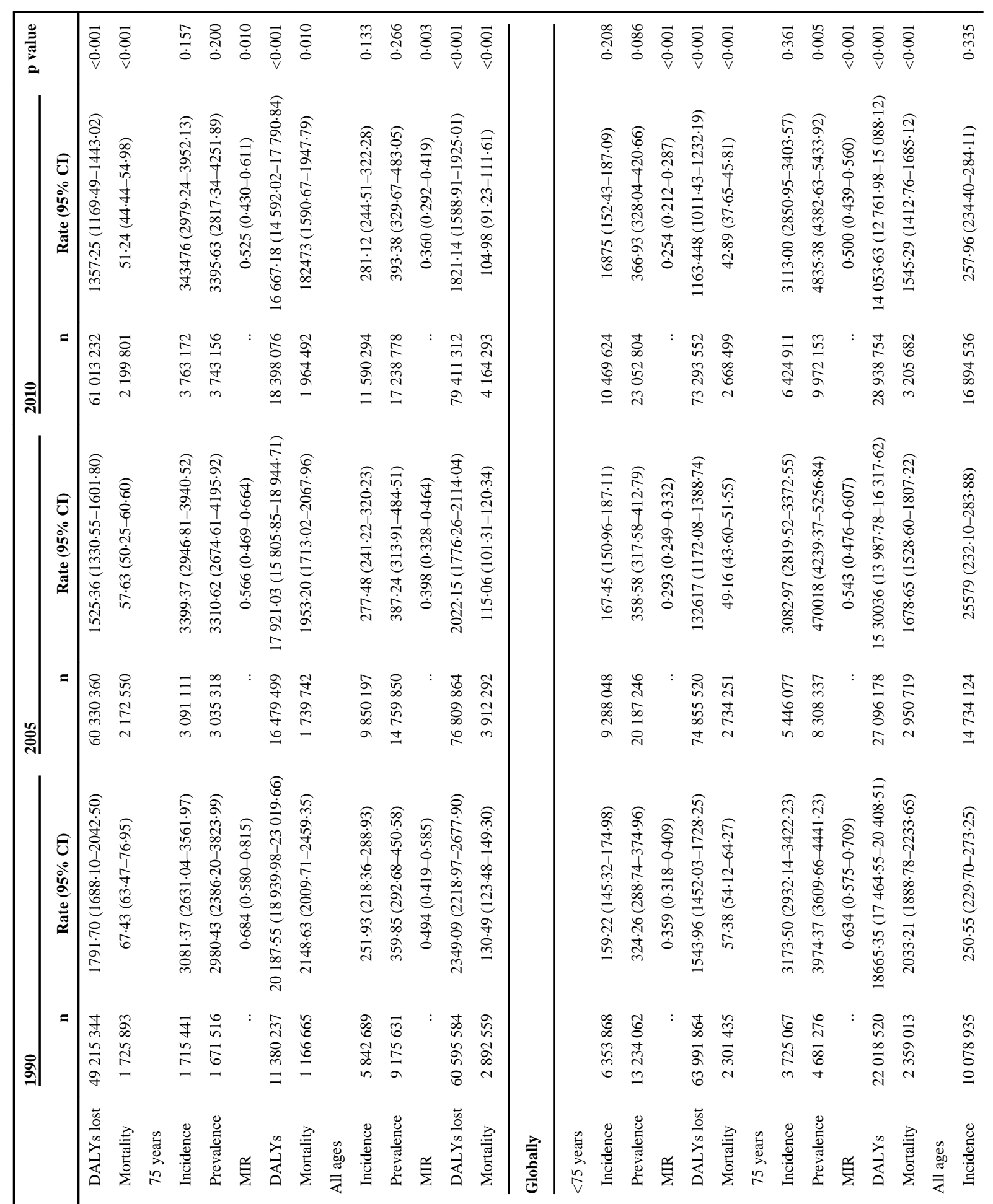


